\title{
Ordinal Sums of De Morgan Triples ${ }^{\dagger}$
}

\author{
I. MEZZOMO ${ }^{1}$ and B. BEDREGAL ${ }^{2}$
}

Received on March 31, 2017 / Accepted on November 14, 2017

\begin{abstract}
In this paper we consider the ordinal sum of the summands of t-norms, t-conorms, and fuzzy negations and prove some new results about them. In particular, we provide some conditions in order to guarantee that when the summands are De Morgan triples their ordinal sums also are De Morgan triple. We also proved that De Morgan laws are preserved for the the action of automorphisms and $N$-duality.
\end{abstract}

Keywords: t-norm, t-conorm, fuzzy negation, De Morgan triples, ordinal sum.

\section{INTRODUCTION}

There are several ways to extend the propositional classical connectives for a set $[0,1]$, but not always these extentions preserve the properties of the classical conectives. Triangular norms (tnorms) and triangular conorms (t-conorms) were first studied by Menger [19] and, Scheweizer and Sklar [26] in probabilistic metric spaces in which triangular inequalities were extended using t-norms and t-conorms theory.

The name triangular norm refers to the fact that in the framework of probabilistic metric spaces t-norms are used to generalize triangle inequality of ordinary metric spaces and t-conorms are dual to t-norms under the order-reversing operation. The defining conditions of the t-norm are exactly those of the partially ordered Abelian monoid on the real unit interval $[0,1]$.

T-norms are used to represent logical conjunction in fuzzy logic and interseption in fuzzy set theory, whereas t-conorms are used to represent logical disjunction in fuzzy logic and union in fuzzy set theory. A t-norm (t-conorm) is a binary operation defined in $[0,1]$, which is commutative, associative, nondecreasing and with neutral element $1(0)$.

In 19th century, De Morgan introduced the De Morgan's laws which in propositional logic and boolean algebra is a pair of transformation rules that are both valid rules of inference. This

\footnotetext{
${ }^{\dagger}$ This paper extends the work published by Proceeding of Fourth Brazilian Conference on Fuzzy Systems (IV CBSF) (2016)

*Corresponding author: Ivan Mezzomo - E-mail: imezzomo@ufersa.edu.br

${ }^{1}$ Centro de Ciências Exatas e Naturais - DCEN, Universidade Federal Rural do Semi-Árido - UFERSA, Mossoró, Rio Grande do Norte, Brazil. E-mail: imezzomo@ufersa.edu.br

2 Departamento de Informática e Matemática Aplicada - DIMAp, Universidade Federal do Rio Grande do Norte - UFRN, Natal, Rio Grande do Norte, Brazil. E-mail: bedregal@dimap.ufrn.br
} 
rules allow the expression of conjunctions and disjunctions purely in terms of each other via negation. In formal language, these rules can be expressed as "the negation of a conjunction is the disjunction of the negations" and "the negation of a disjunction is the conjunction of the negations". De Morgan's laws are also apply in the more general context of Boolean algebra and, in particular, in the Boolean algebra of set theory. In fuzzy logic, the triples formed by a t-norm, $\mathrm{t}$-conorm and standard complement is called De Morgan triples if it fulfills De Morgan laws. De Morgan triples were first introduced by Zadeh in 1965.

In this contribuition, our aim is characterize the ordinal sum of the summands $\left(a_{i}, b_{i}, N_{i}\right)$ where $\left(N_{i}\right)_{i \in I}$ are a family of fuzzy negations such that $N_{i} \geq N_{S}$ and (]$a_{i}, b_{i}[)_{i \in I}$ a family of nonempty, pairwise disjoint open subintervals of $[0,1]$, and prove that the function $N$ is a fuzzy negation. In addition, we prove if $\left(T_{i}, S_{i}, N_{i}\right)$ is a De Morgan triple satisfy some specific conditions, then $(T, S, N)$ is a semi De Morgan triple.

This paper is organized as follows: Section 2 provides a review of the most important subjects for this work, such as t-norms, t-conorms, fuzzy negations, automorphisms and De Morgan triples. Section 3 contains some results based in [20], using definitions introduced in previous section. Section 4, we consider the notion of ordinal sum with respect to t-norms and t-conorms defined in [17] and characterize the ordinal sum with respect to fuzzy negations. In section 5, we provide some final remarks and furture works.

\section{PRELIMINARIES}

In this section, we will briefly review some basic concepts which are necessary for the development of this paper. The definitions and additional results can be found in [1], [2], [6], [4], [10], [9],[12], [15], [17].

\section{1 t-norms, t-conorms and fuzzy negations}

Definition 2.1. A function $T:[0,1]^{2} \rightarrow[0,1]$ is a $t$-norm if, for all $x, y, z \in[0,1]$, the following axioms are satisfied:

1. Commutative: $T(x, y)=T(y, x)$;

2. Associative: $T(x, T(y, z))=T(T(x, y), z)$;

3. Monotonic: If $x \leq y$, then $T(x, z) \leq T(y, z)$;

4. One identity: $T(x, 1)=x$.

A t-norm $T$ is called positive if satifies the condiction: $T(x, y)=0$ iff $x=0$ or $y=0$.

Example 2.1. Some examples of $t$-norms:

1. Gödel t-norm: $T_{G}(x, y)=\min (x, y)$; 
2. Product t-norm: $T_{P}(x, y)=x \cdot y$;

3. Lukasiewicz t-norm: $T_{L}(x, y)=\max (0, x+y-1)$;

4. Drastic t-norm:

$$
T_{D}(x, y)= \begin{cases}0 & \text { if }(x, y) \in\left[0,1\left[^{2}\right.\right. \\ \min (x, y) & \text { otherwise }\end{cases}
$$

Definition 2.2. A function $S:[0,1]^{2} \rightarrow[0,1]$ is a $t$-conorm if, for all $x, y, z \in[0,1]$, the following axioms are satisfied:

1.Commutative: $S(x, y)=S(y, x)$;

2. Associative: $S(x, S(y, z))=S(S(x, y), z)$;

3. Monotonic: If $x \leq y$, then $S(z, x) \leq S(z, y)$;

4. Zero identity: $S(x, 0)=x$.

A t-conorm $S$ is called positive if satifies the condiction: $S(x, y)=1$ iff $x=1$ or $y=1$.

Example 2.2. Some examples of t-conorms:

1. Gödel t-conorm: $S_{G}(x, y)=\max (x, y)$;

2. Probabilistic sum: $S_{P}(x, y)=x+y-x \cdot y$;

3. Lukasiewicz t-conorm: $S_{L}(x, y)=\min (x+y, 1)$;

\section{Drastic sum:}

$$
S_{D}(x, y)= \begin{cases}1 & \text { if }(x, y) \in] 0,1]^{2} \\ \max (x, y) & \text { otherwise }\end{cases}
$$

A function $N:[0,1] \rightarrow[0,1]$ is a fuzzy negation if

$\mathrm{N} 1: N(0)=1$ and $N(1)=0$;

N2: Decreasing: If $x \leq y$, then $N(x) \geq N(y)$, for all $x, y \in[0,1]$. 
A fuzzy negations $N$ is strict if it is continuous and strictly decreasing, i.e., $N(x)<N(y)$ when $y<x$. A fuzzy negations $N$ that satisfying the condition N3 is called strong.

N3: Involutive: $N(N(x))=x$ for each $x \in[0,1]$.

A fuzzy negation is called crisp if satisfies N4

N4: Crisp: For all $x \in[0,1], N(x) \in\{0,1\}$.

Example 2.3. Some examples of fuzzy negations:

1. Standard negation: $N_{S}(x)=1-x$;

2. Strict non-strong negation: $N_{S^{2}}(x)=1-x^{2}$;

\section{Bottom negation:}

$$
N_{\perp}(x)=\left\{\begin{array}{lll}
0 & \text { if } & x>0 \\
1 & \text { if } & x=0
\end{array}\right.
$$

\section{Top negation:}

$$
N_{\top}(x)=\left\{\begin{array}{lll}
0 & \text { if } & x=1 \\
1 & \text { if } & x<1
\end{array}\right.
$$

In [13], Dimuro et.al. define $N_{\alpha}$ and $N^{\alpha}$ as

$$
\begin{gathered}
N_{\alpha}(x)=\left\{\begin{array}{lll}
0 & \text { if } & x>\alpha \\
1 & \text { if } & x \leq \alpha
\end{array}\right. \\
N^{\alpha}(x)=\left\{\begin{array}{lll}
0 & \text { if } & x \geq \alpha \\
1 & \text { if } & x<\alpha
\end{array}\right.
\end{gathered}
$$

In [13] is proved that a fuzzy negation $N$ is crisp iff there exists a $\alpha \in[0,1]$ such that $N=N_{\alpha}$ or $N=N^{\alpha}$.

Definition 2.3. [3, Definitions 2.3.8 and 2.3.14] Let $T$ be a t-norm, $S$ be a t-conorm and $N$ be a fuzzy negation. The pair $(T, N)$ satisfies the law of contradiction if

$$
T(x, N(x))=0, \forall x \in[0,1]
$$

The pair $(S, N)$ satisfies the law of excluded middle if

$$
S(x, N(x))=1, \forall x \in[0,1]
$$

Note that: 
1. If $N$ is strong then it has an inverse $N^{-1}$ which also is a strict fuzzy negation;

2. If $N$ is strong then $N$ is strict.

The supremum and infimum of the t-norms $T_{1}$ and $T_{2}$ is defined as

$$
\begin{gathered}
T_{1} \wedge T_{2}(x, y)=\min \left\{T_{1}(x, y), T_{2}(x, y)\right\} \\
T_{1} \vee T_{2}(x, y)=\max \left\{T_{1}(x, y), T_{2}(x, y)\right\} .
\end{gathered}
$$

Similarly, we define the supremum and infimum of the t-conorms $S_{1}$ and $S_{2}$.

Proposition 2.1. Let $T_{1}$ and $T_{2}$ be t-norms. Then, $T_{1} \wedge T_{2}$ and $T_{1} \vee T_{2}$ are symmetric, increasing and have 1 as neutral element.

Proof. Let $T_{1}$ and $T_{2}$ be t-norms. Then, for all $x, y, z \in[0,1]$,

1. Symmetry:

$$
\begin{aligned}
T_{1} \wedge T_{2}(x, y) & =\min \left\{T_{1}(x, y), T_{2}(x, y)\right\} \\
& =\min \left\{T_{1}(y, x), T_{2}(y, x)\right\} \\
& =T_{1} \wedge T_{2}(y, x) .
\end{aligned}
$$

2. Monotonicity: If $x \leq y$ then, since $T$ is a t-norm, $T_{1}(x, z) \leq T_{1}(y, z)$ and $T_{2}(x, z) \leq T_{2}(y, z)$. Thus, $\min \left\{T_{1}(x, z), T_{2}(x, z)\right\} \leq \min \left\{T_{1}(y, z), T_{2}(y, z)\right\}$. Therefore, $T_{1} \wedge T_{2}(x, z) \leq T_{1} \wedge T_{2}(y, z)$.

3. Border Condition: For all $x \in[0,1]$, we have that

$$
\begin{aligned}
T_{1} \wedge T_{2}(x, 1) & =\min \left\{T_{1}(x, 1), T_{2}(x, 1)\right\} \\
& =\min \{x, x\} \\
& =x
\end{aligned}
$$

Analogously we prove that $T_{1} \vee T_{2}$ is symmetric, increasing and have 1 as neutral element.

Notice that, not always $T_{1} \wedge T_{2}$ and $T_{1} \vee T_{2}$ are associative and therefore, t-norms.

Proposition 2.2. Let $S_{1}$ and $S_{2}$ be t-conorms. Then, $S_{1} \wedge S_{2}$ and $S_{1} \vee S_{2}$ are symmetric, increasing and have 0 as neutral element.

Proof. Analogous from Proposition 2.1.

Definition 2.4. Let $T$ be a t-norm, $S$ be a t-conorm and $N$ be a strict fuzzy negation. $T_{N}$ is the $N$-dual of $T$ if, for all $x, y \in[0,1], T_{N}(x, y)=N^{-1}(T(N(x), N(y)))$. Similarly, $S_{N}$ is the $N$-dual of $S$ if, for all $x, y \in[0,1], S_{N}(x, y)=N^{-1}(S(N(x), N(y)))$. 
Proposition 2.3. Let $T$ be a $t$-norm, $S$ be a $t$-conorm and $N$ be a strict fuzzy negation. Then, $T_{N}$ is a t-conorm and $S_{N}$ is a $t$-norm.

Proof. Let $T$ be a t-norm and $N$ be a fuzzy negation. Then, for all $x, y, z \in[0,1]$,

1. Symmetry:

$$
\begin{aligned}
T_{N}(x, y) & =N^{-1}(T(N(x), N(y))) \\
& =N^{-1}(T(N(y), N(x))) \\
& =T_{N}(y, x) .
\end{aligned}
$$

2. Associativity:

$$
\begin{aligned}
T_{N}\left(x, T_{N}(y, z)\right) & =N^{-1}\left(T\left(N(x), N\left(N^{-1}(T(N(y), N(z)))\right)\right)\right) \\
& =N^{-1}(T(N(x), T(N(y), N(z)))) \\
& =N^{-1}(T(T(N(x), N(y)), N(z))) \\
& =N^{-1}\left(T\left(N\left(N^{-1}(T(N(x), N(y))), N(z)\right)\right)\right) \\
& =T_{N}\left(T_{N}(x, y), z\right)
\end{aligned}
$$

3. Monotonicity: If $y \leq z$, then $N(z) \leq N(y)$. Since $T$ is a t-norm, then $T(N(x), N(z)) \leq$ $T(N(x), N(y))$. Thus, $\quad N^{-1}(T(N(x), N(y))) \leq N^{-1}(T(N(x), N(z)))$. Therefore, $T_{N}(x, y) \leq T_{N}(x, z)$.

4. Border Condition: For all $x \in[0,1]$, we have that

$$
\begin{aligned}
T_{N}(x, 0) & =N^{-1}(T(N(x), N(0))) \\
& =N^{-1}(T(N(x), 1)) \\
& =N^{-1}(N(x)) \\
& =x .
\end{aligned}
$$

Therefore, $T_{N}$ is a t-conorm. Analogously we prove that $S_{N}$ is a t-norm.

Definition 2.5. A function $\rho:[0,1] \rightarrow[0,1]$ is an automorphism if it is bijective and increasing, i.e., for each $x, y \in[0,1]$, if $x \leq y$, then $\rho(x) \leq \rho(y)$.

According with [9, Definition 0], a function $\rho: U \rightarrow U$ is an automorphism if it is continuous, strictly increasing and verifies the boundary conditions $\rho(0)=0$ and $\rho(1)=1$, i.e., if it is an increasing bijection on $U$, meaning that for each $x, y \in[0,1]$, if $x \leq y$, then $\rho(x) \leq \rho(y)$. The set of all automorphisms on $[0,1]$ will be denoted by $A u t([0,1])$. 
Automorphisms are closed under composition, i.e., if $\rho, \rho^{\prime} \in \operatorname{Aut}([0,1])$, then $\rho \circ \rho^{\prime} \in A u t([0,1])$, where $\rho \circ \rho^{\prime}(x)=\rho\left(\rho^{\prime}(x)\right)$. In addition, the inverse $\rho^{-1}$ of an automorphism $\rho$ is also an automorphism.

The function $T^{\rho}\left(S^{\rho}\right)$, called as the $\rho$-conjugate of a t-(co)norm $T(S)$, is obtained by action of $\rho \in \operatorname{Aut}([0,1])$ on $T(S)$ and defined in the following:

$$
\begin{aligned}
T^{\rho}(x, y) & =\rho^{-1}(T(\rho(x), \rho(y))), \\
S^{\rho}(x, y) & =\rho^{-1}(S(\rho(x), \rho(y))), \forall x, y \in[0,1] .
\end{aligned}
$$

\subsection{De Morgan triples}

According to [17], the triple $(T, S, N)$ where $T$ is a t-norm, $S$ is a t-conorm and $N$ a fuzzy negation is called De Morgan triples if satisfies the following conditions:

$$
\begin{aligned}
& T(x, y)=N(S(N(x), N(y))) ; \\
& S(x, y)=N(T(N(x), N(y))),
\end{aligned}
$$

which naturally imply that $N$ is a strong fuzzy negation [12].

There are several different notions of De Morgan triples as we can see in [7], [11], [16], [18], [21], [22]. In this paper we will use the definition of De Morgan triples laws as done in [12], [18], [22], [29] which not implies in involution of the fuzzy negation. Thus,

Definition 2.6. Let $T$ is a t-norm, $S$ a t-conorm, $N$ a fuzzy negation. Then $(T, S, N)$ is a De Morgan triple if, for each $x, y \in[0,1]$,

$$
\begin{aligned}
& N(T(x, y))=S(N(x), N(y)) ; \\
& N(S(x, y))=T(N(x), N(y)) .
\end{aligned}
$$

Example 2.4. $\left(T_{G}, S_{G}, N_{S}\right),\left(T_{P}, S_{P}, N_{\perp}\right)$ and $\left(T_{L}, S_{L}, N_{S}\right)$ are examples of De Morgan triples.

Definition 2.7. $(T, S, N)$ is a semi De Morgan triple if satify the Eq. (2.5) for each $x \in[0,1]$ or (2.6) for each $x \in[0,1]$.

\section{RESULTS ABOUT DE MORGAN TRIPLES}

In this section, we will prove some propositions using definitions introduced on the previous section. The results of this section is based in [20].

Proposition 3.4. Let $T$ be a t-norm, $S$ be a t-conorm and $\alpha \in[0,1]$. If, $T(x, y)>\alpha \Leftrightarrow x, y>\alpha$ and $S(x, y) \leq \alpha \Leftrightarrow x, y \leq \alpha$, then $\left(T, S, N_{\alpha}\right)$ is a De Morgan triple. 
Proof. Suppose that $x, y>\alpha$, then $T(x, y)>\alpha$ and $N_{\alpha}(T(x, y))=0$. On the other hand, $S\left(N_{\alpha}(x), N_{\alpha}(y)\right)=S(0,0)=0 ;$

If $x \leq \alpha$, then $T(x, y) \leq \alpha$ and therefore, $N_{\alpha}(T(x, y))=1$. On the other hand, $S\left(N_{\alpha}(x), N_{\alpha}(y)\right)=$ $S\left(1, N_{\alpha}(y)\right)=1$;

If $y \leq \alpha$, the proof is analogous. Therefore, $N_{\alpha}(T(x, y))=S\left(N_{\alpha}(x), N_{\alpha}(y)\right)$.

Now, we will prove that $N_{\alpha}(S(x, y))=T\left(N_{\alpha}(x), N_{\alpha}(y)\right)$. Suppose that $x, y \leq \alpha$, then $S(x, y) \leq \alpha$ and $N_{\alpha}(S(x, y))=1$. On the other hand, $T\left(N_{\alpha}(x), N_{\alpha}(y)\right)=T(1,1)=1$;

If $x>\alpha$, then $S(x, y)>\alpha$ and therefore, $N_{\alpha}(S(x, y))=0$. On the other hand, $T\left(N_{\alpha}(x), N_{\alpha}(y)\right)=$ $T\left(0, N_{\alpha}(y)\right)=0$;

If $y>\alpha$, the proof is analogous. Therefore, $N_{\alpha}(S(x, y))=T\left(N_{\alpha}(x), N_{\alpha}(y)\right)$.

Therefore, $\left(T, S, N_{\alpha}\right)$ is a De Morgan triple.

Corollary 3.0. Let $T$ be a positive $t$-norm and $S$ be a positive $t$-conorm. Then, $\left(T, S, N_{\perp}\right)$ is a De Morgan triple.

Proof. Analogous from Proposition 3.4.

Proposition 3.5. Let $T$ be a t-norm, $S$ be a t-conorm and $\alpha \in[0,1]$. If, $T(x, y) \geq \alpha \Leftrightarrow x, y \geq \alpha$ and $S(x, y)<\alpha \Leftrightarrow x, y<\alpha$, then $\left(T, S, N^{\alpha}\right)$ is a De Morgan triple.

Proof. Analogous from Proposition 3.4.

Corollary 3.0. Let $T$ be a positive $t$-norm and $S$ be a positive $t$-conorm. Then, $\left(T, S, N_{T}\right)$ is a De Morgan triple.

Proof. Analogous from Proposition 3.4.

Proposition 3.6. Let $(T, S, N)$ be a De Morgan triple. If $N$ is strict then $\left(T, S, N^{-1}\right)$ is also De Morgan triple.

Proof. Let $x, y \in[0,1]$, then

$$
\begin{aligned}
N^{-1}(T(x, y)) & =N^{-1}\left(T\left(N\left(N^{-1}(x)\right), N\left(N^{-1}(y)\right)\right)\right) \\
& =N^{-1}\left(N\left(S\left(N^{-1}(x), N^{-1}(y)\right)\right)\right) \\
& =S\left(N^{-1}(x), N^{-1}(y)\right) .
\end{aligned}
$$

Analogously we prove that $N^{-1}(S(x, y))=T\left(N^{-1}(x), N^{-1}(y)\right)$.

The following lemmas will give us important results for to prove the propositions envolving contradiction law and law of excluded middle.

Lemma 3.1. Let $T$ be a t-norm and $N$ be a strict fuzzy negation. $(T, N)$ satisfies $(L C)$ iff $\left(T, N^{-1}\right)$ satisfies $(L C)$. 
Proof. Since $N$ is strict then it has an inverse strict fuzzy negation $N^{-1}$.

$(\Rightarrow)$ Since $(T, N)$ satisfies (LC), then $T\left(N(x), N^{-1}(N(x))\right)=0$, for all $x \in[0,1]$. So, for all $x \in$ $[0,1], T(x, N(x))=T(N(x), x)=T\left(N(x), N^{-1}(N(x))\right)=0$.

$(\Leftarrow)$ Analogous.

Lemma 3.2. Let $S$ be a t-conorm and $N$ be a strict fuzzy negation. $(S, N)$ satisfies (LEM) iff $\left(S, N^{-1}\right)$ satisfies (LEM).

Proof. Analogous from Lemma 3.1.

Proposition 3.7. Let $(T, S, N)$ be a semi De Morgan triple with respect the Eq. (2.5) and $N$ be a strict fuzzy negation. If $(T, N)$ satisfies $(L C)$ then $(S, N)$ satisfies $(L E M)$.

Proof. From Lemma 3.1, we have $S\left(N^{-1}(x), x\right)=1, \forall x \in[0,1]$. Since $(T, S, N)$ satisfies Eq. (2.5), we obtain that $0=N\left(S\left(N^{-1}(x), x\right)\right)=T(x, N(x))$ for all $x \in[0,1]$.

Proposition 3.8. Let $(T, S, N)$ be a semi De Morgan triple with respect the Eq. (2.6) and $N$ be a strict fuzzy negation. If $(S, N)$ satisfies (LEM) then $(T, N)$ satisfies $(L C)$.

Proof. From Lemma 3.2, we have $T\left(x, N^{-1}(x)\right)=0, \forall x \in[0,1]$. Since $(T, S, N)$ satisfies Eq. (2.6), we obtain that $1=N\left(T\left(x, N^{-1}(x)\right)\right)=S(N(x), x)$ for all $x \in[0,1]$.

Corollary 3.0. Let $(T, S, N)$ be a De Morgan triple and $N$ be a strict fuzzy negation. Then, $(T, N)$ satisfies (LC) iff $(S, N)$ satisfies (LEM).

Proof. Straighforward from Propositions 3.7 and 3.8.

Proposition 3.9. Let $(T, S, N)$ be a De Morgan triple. If $N$ is strict then $\left(S_{N}, T_{N}, N\right)=(T, S, N)$.

Proof. Since $N$ is strict, then $S_{N}(x, y)=N^{-1}(S(N(x), N(y)))=N^{-1}(N(T(x, y)))=T(x, y)$. Analogously we prove that $T_{N}(x, y)=S(x, y)$.

Proposition 3.10. Let $(T, S, N)$ be a De Morgan triple. If $N$ is strict, then $\left(S_{N}, T_{N^{-1}}, N\right)$ and $\left(S_{N^{-1}}, T_{N}, N\right)$ are semi De Morgan triple.

Proof. Let $N$ be a strict fuzzy negation and $x, y \in[0,1]$. Then

$$
\begin{aligned}
N\left(S_{N}(x, y)\right) & =N\left(N^{-1}(S(N(x), N(y)))\right) \\
& =S(N(x), N(y)) \\
& =N(T(x, y)) \\
& =N\left(T\left(N^{-1}(N(x)), N^{-1}(N(y))\right)\right) \\
& =T_{N^{-1}}(N(x), N(y)) .
\end{aligned}
$$

Thus, $\left(S_{N}, T_{N^{-1}}, N\right)$ satisfies the Eq. (2.6) and therefore, it is a semi De Morgan triple. Analogously, we prove that $\left(S_{N^{-1}}, T_{N}, N\right)$ satisfies Eq. (2.5). 
Proposition 3.11. Let $\left(T_{1}, S_{1}, N\right)$ and $\left(T_{2}, S_{2}, N\right)$ be De Morgan triples. Then, $T_{1} \leq T_{2}$ iff $S_{1} \geq S_{2}$.

Proof. $(\Rightarrow)$ Let $T_{1} \leq T_{2}$ and $x, y \in[0,1]$. Then, $N\left(S_{1}(x, y)\right)=T_{1}(N(x), N(y)) \leq T_{2}(N(x), N(y))=$ $N\left(S_{2}(x, y)\right)$. Therefore, $S_{1}(x, y) \geq S_{2}(x, y)$.

$(\Leftarrow)$ Analogous.

Proposition 3.12. Let $\left(T_{1}, S_{1}, N\right)$ and $\left(T_{2}, S_{2}, N\right)$ be De Morgan triples. If $T_{1} \wedge T_{2}$ and $S_{1} \vee S_{2}$ are t-norm and t-conorm, respectively, then $\left(T_{1} \wedge T_{2}, S_{1} \vee S_{2}, N\right)$ is a De Morgan triple. Dually, if $T_{1} \vee T_{2}$ and $S_{1} \wedge S_{2}$ are $t$-norm, $t$-conorm, respectively, then $\left(T_{1} \vee T_{2}, S_{1} \wedge S_{2}, N\right)$ is a De Morgan triple.

Proof. Let $x, y \in[0,1]$. Then,

$$
\begin{aligned}
N\left(T_{1} \wedge T_{2}(x, y)\right) & =N\left(\min \left\{T_{1}(x, y), T_{2}(x, y)\right\}\right) \\
& =\max \left\{N\left(T_{1}(x, y)\right), N\left(T_{2}(x, y)\right)\right\} \\
& =\max \left\{S_{1}(N(x), N(y)), S_{2}(N(x), N(y))\right\} \\
& =S_{1} \vee S_{2}(N(x), N(y)) .
\end{aligned}
$$

Analogously we prove that $N\left(S_{1} \vee S_{2}(x, y)\right)=T_{1} \wedge T_{2}(N(x), N(y))$. Therefore, $\left(T_{1} \wedge T_{2}, S_{1} \vee S_{2}, N\right)$ is a De Morgan triple.

Dually, we prove that $\left(T_{1} \vee T_{2}, S_{1} \wedge S_{2}, N\right)$ is a De Morgan triple.

Proposition 3.13. Let $(T, S, N)$ be a De Morgan triple. If $N$ is strict then $T=S_{N}=S_{N^{-1}}$ and $S=T_{N}=T_{N^{-1}}$.

Proof. Since $N$ is strict, then $S_{N}(x, y)=N^{-1}(S(N(x), N(y)))=N^{-1}(N(T(x, y)))=T(x, y)$ and $T(x, y)=T\left(N\left(N^{-1}(x)\right), N\left(N^{-1}(y)\right)\right)=N\left(S\left(N^{-1}(x), N^{-1}(y)\right)\right)=S_{N^{-1}}(x, y)$. Therefore, $T=$ $S_{N}=S_{N^{-1}}$. Analogously we prove that $S=T_{N}=T_{N^{-1}}$.

Now, using the notion of automorphism $\rho$ from t-norms, t-conorms and fuzzy negation, we show that the triple $\left(T^{\rho}, S^{\rho}, N^{\rho}\right)$ is a De Morgan triple.

Proposition 3.14. Let $(T, S, N)$ be a De Morgan triple and $\rho$ be an automorphism. Then, $\left(T^{\rho}, S^{\rho}, N^{\rho}\right)$ is a De Morgan triple.

Proof. Let $x, y \in[0,1]$, then

$$
\begin{aligned}
N^{\rho}\left(T^{\rho}(x, y)\right) & =N^{\rho}\left(\rho^{-1}(T(\rho(x), \rho(y)))\right) \\
& =\rho^{-1}\left(N\left(\rho\left(\rho^{-1}(T(\rho(x), \rho(y)))\right)\right)\right) \\
& =\rho^{-1}(N(T(\rho(x), \rho(y)))) \\
& =\rho^{-1}(S(N(\rho(x)), N(\rho(y)))) \\
& =\rho^{-1}\left(S\left(\rho\left(\rho^{-1}(N(\rho(x)))\right), \rho\left(\rho^{-1}(N(\rho(y)))\right)\right)\right) \\
& =S^{\rho}\left(\rho^{-1}(N(\rho(x))), \rho^{-1}(N(\rho(y)))\right) \\
& =S^{\rho}\left(N^{\rho}(x), N^{\rho}(y)\right) .
\end{aligned}
$$


Analogously we prove that $N^{\rho}\left(S^{\rho}(x, y)\right)=T^{\rho}\left(N^{\rho}(x), N^{\rho}(y)\right)$.

\section{ORDINAL SUMS OF DE MORGAN TRIPLES}

In this section, we consider the notion of ordinal sum with respect to t-norms and t-conorms defined in [17] and characterize the ordinal sum with respect to fuzzy negations.

Proposition 4.15. [17] Let $\left(T_{i}\right)_{i \in I}$ be a family of $t$-norms and (]$a_{i}, b_{i}[)_{i \in I}$ be a family of nonempty, pairwise disjoint open subintervals of $[0,1]$. Then the function $T:[0,1]^{2} \rightarrow[0,1]$ defined by

$$
T(x, y)= \begin{cases}a_{i}+\left(b_{i}-a_{i}\right) T_{i}\left(\frac{x-a_{i}}{b_{i}-a_{i}}, \frac{y-a_{i}}{b_{i}-a_{i}}\right) & \text { if }(x, y) \in\left[a_{i}, b_{i}\right]^{2}, \\ \min (x, y) & \text { otherwise. }\end{cases}
$$

is a $t$-norm which is called the ordinal sum of the summands $\left(a_{i}, b_{i}, T_{i}\right), i \in I$.

Proposition 4.16. [17] Let $\left(S_{i}\right)_{i \in I}$ be a family of t-conorms and (]$a_{i}, b_{i}[)_{i \in I}$ be a family of nonempty, pairwise disjoint open subintervals of $[0,1]$. Then the function $S:[0,1]^{2} \rightarrow[0,1]$ defined by

$$
S(x, y)= \begin{cases}a_{i}+\left(b_{i}-a_{i}\right) S_{i}\left(\frac{x-a_{i}}{b_{i}-a_{i}}, \frac{y-a_{i}}{b_{i}-a_{i}}\right) & \text { if }(x, y) \in\left[a_{i}, b_{i}\right]^{2}, \\ \max (x, y) & \text { otherwise. }\end{cases}
$$

is a $t$-conorm which is called the ordinal sum of the summands $\left(a_{i}, b_{i}, S_{i}\right), i \in I$.

Definition 4.8. Let $\left(N_{i}\right)_{i \in I}$ be a family of fuzzy negations and (]$a_{i}, b_{i}[)_{i \in I}$ be a family of nonempty, pairwise disjoint open subintervals of $[0,1]$. Then the function $N:[0,1] \rightarrow[0,1]$ defined by

$$
N(x)= \begin{cases}\left(1-b_{i}\right)+\left(b_{i}-a_{i}\right) N_{i}\left(\frac{x-a_{i}}{b_{i}-a_{i}}\right) & \text { if } x \in\left[a_{i}, b_{i}\right], \\ N_{S}(x) & \text { otherwise }\end{cases}
$$

is called of the ordinal sum of the summands $\left(a_{i}, b_{i}, N_{i}\right), i \in I$.

Lemma 4.3. Let (]$a_{i}, b_{i}[)_{i \in I}$ be a family of nonempty, pairwise disjoint open subintervals of $[0,1]$, $\left(N_{i}\right)_{i \in I}$ be a family of fuzzy negations and $N$ the ordinal sum $N$ of the summands $\left(a_{i}, b_{i}, N_{i}\right), i \in I$. Then,

1. If $x \in\left[a_{i}, b_{i}\right]$ for some $i \in I$ then $N(x) \in\left[1-b_{i}, 1-a_{i}\right]$.

2. If $x \notin \bigcup_{i \in I}\left[a_{i}, b_{i}\right]$ then $N(x) \notin \bigcup_{i \in I}\left[1-b_{i}, 1-a_{i}\right]$.

Proof. If $x \in\left[a_{i}, b_{i}\right]$ for some $i \in I$ then by Eq. (4.3) and because $N_{i}$ is a fuzzy negation, we have that

$$
\begin{aligned}
& N(x) \in\left[\left(1-b_{i}\right)+\left(b_{i}-a_{i}\right) N_{i}\left(\frac{b_{i}-a_{i}}{b_{i}-a_{i}}\right),\left(1-b_{i}\right)+\left(b_{i}-a_{i}\right) N_{i}\left(\frac{a_{i}-a_{i}}{b_{i}-a_{i}}\right)\right] \\
= & {\left[\left(1-b_{i}\right)+\left(b_{i}-a_{i}\right) N_{i}(1),\left(1-b_{i}\right)+\left(b_{i}-a_{i}\right) N_{i}(0)\right]=\left[1-b_{i}, 1-a_{i}\right] . }
\end{aligned}
$$


If $x \notin \bigcup_{i \in I}\left[a_{i}, b_{i}\right]$ then by Eq. (4.3), $N(x)=1-x$. Suppose, that $1-x \in\left[1-b_{i}, 1-a_{i}\right]$ for some $i \in I$, then, trivially, $x \in\left[a_{i}, b_{i}\right]$ which is a contradiction. Therefore, $N(x) \notin \bigcup_{i \in I}\left[1-b_{i}, 1-a_{i}\right]$.

Proposition 4.17. Let (]$a_{i}, b_{i}[)_{i \in I}$ be a family of nonempty, pairwise disjoint open subintervals of $[0,1]$ and $\left(N_{i}\right)_{i \in I}$ be a family of fuzzy negations. Then the ordinal sum $N$ of the summands $\left(a_{i}, b_{i}, N_{i}\right), i \in I$ is a fuzzy negation.

Proof. If there exists $i \in I$ such that $a_{i}=0$, then by Eq. (4.3), $N(0)=\left(1-b_{i}\right)+b_{i} N_{i}(0)=1$. On the other hand, if there exists no $i \in I$ such that $a_{i}=0$, then by Eq. (4.3), $N(0)=N_{S}(0)=1$. In analogous way, we can prove that $N(1)=0$.

If $x \leq y$ then we have the following cases:

Case 1: If $x, y \in\left[a_{i}, b_{i}\right]$ for some $i \in I$, then $\frac{x-a_{i}}{b_{i}-a_{i}} \leq \frac{y-a_{i}}{b_{i}-a_{i}}$ and therefore $N_{i}\left(\frac{y-a_{i}}{b_{i}-a_{i}}\right) \leq$ $N_{i}\left(\frac{x-a_{i}}{b_{i}-a_{i}}\right)$. So, Eq. (4.3), $N(y) \leq N(x)$.

Case 2: If $x \in\left[a_{i}, b_{i}\right]$ and $y \in\left[a_{j}, b_{j}\right]$ for some $i, j \in I$ such that $i \neq j$ then $a_{i}<b_{i}<a_{j}<b_{j}$. So, by Lemma 4.3, $N(y) \in\left[1-a_{j}, 1-b_{j}\right]$ and $N(x) \in\left[1-b_{i}, 1-a_{i}\right]$. Thus, since $1-b_{j}<1-a_{i}$, then $N(y)<N(x)$.

Case 3: If $x \in\left[a_{i}, b_{i}\right]$ for some $i \in I$ and $y \notin \bigcup_{j \in I}\left[a_{j}, b_{j}\right]$, then $a_{i} \leq x$ and therefore $1-y \leq 1-a_{i}$. Since, by Lemma 4.3,1-a $\leq N(x)$ and by Eq. (4.3) $N(y)=1-y$, then follows that $N(y) \leq N(x)$.

Case 4: If $x \notin \bigcup_{j \in I}\left[a_{j}, b_{j}\right]$ and $y \in\left[a_{i}, b_{i}\right]$ for some $i \in I$, then $x<b_{i}$. So, by Eq. (4.3) $N(x)=1-x$ and therefore, by Lemma 4.3, $N(y) \leq 1-b_{i} \leq 1-x=N(x)$.

Case 5: If $x, y \notin \bigcup_{i \in I}\left[a_{i}, b_{i}\right]$ then by Eq. (4.3), $N(y)=1-y \leq 1-x=N(x)$.

Theorem 4.1. Let $T, S$ and $N$ be the ordinal sum of the summands $\left(a_{i}, b_{i}, T_{i}\right),\left(1-b_{i}, 1-a_{i}, S_{i}\right)$, $\left(a_{i}, b_{i}, N_{i}\right), i \in I$, respectively. If, $N$ is a fuzzy negation and for all $i \in I,\left(T_{i}, S_{i}, N_{i}\right)$ is a De Morgan triple, then $(T, S, N)$ is a semi De Morgan triple which satisfies Eq. (2.5).

Proof. Let $x, y \in[0,1]$. If $(x, y) \notin\left[a_{i}, b_{i}\right]^{2}$ for all $i \in I$, then by Lemma 4.3, $(N(x), N(y)) \notin[1-$ $\left.b_{i}, 1-a_{i}\right]^{2}$ for all $i \in I$. So, $N(T(x, y))=N(\min (x, y))=\max (N(x), N(y))=S(N(x), N(y))$. 
If $(x, y) \in\left[a_{i}, b_{i}\right]^{2}$ for some $i \in I$ then, $T(x, y) \in\left[a_{i}, b_{i}\right]$ and by Propositions 4.15, 4.16 and 4.17, we have that

$$
\begin{aligned}
N(T(x, y)) & =\left(1-b_{i}\right)+\left(b_{i}-a_{i}\right) N_{i}\left(\frac{T(x, y)-a_{i}}{b_{i}-a_{i}}\right) \\
& =\left(1-b_{i}\right)+\left(b_{i}-a_{i}\right) N_{i}\left(\frac{a_{i}+\left(b_{i}-a_{i}\right) T_{i}\left(\frac{x-a_{i}}{b_{i}-a_{i}}, \frac{y-a_{i}}{b_{i}-a_{i}}\right)-a_{i}}{b_{i}-a_{i}}\right) \\
& =\left(1-b_{i}\right)+\left(b_{i}-a_{i}\right) N_{i}\left(T_{i}\left(\frac{x-a_{i}}{b_{i}-a_{i}}, \frac{y-a_{i}}{b_{i}-a_{i}}\right)\right) \\
& =\left(1-b_{i}\right)+\left(b_{i}-a_{i}\right) S_{i}\left(N_{i}\left(\frac{x-a_{i}}{b_{i}-a_{i}}\right), N_{i}\left(\frac{y-a_{i}}{b_{i}-a_{i}}\right)\right) \\
& =\left(1-b_{i}\right)+\left(b_{i}-a_{i}\right) S_{i}\left(\frac{N(x)-\left(1-b_{i}\right)}{b_{i}-a_{i}}, \frac{N(y)-\left(1-b_{i}\right)}{b_{i}-a_{i}}\right) \\
& =S(N(x), N(y)) .
\end{aligned}
$$

Theorem 4.2. Let $T$ and $S$ be the ordinal sum of the summands $\left(a_{i}, b_{i}, T_{i}\right)$ and $\left(1-b_{i}, 1-a_{i}, S_{i}\right)$, $i \in I$, respectively. If, for all $i \in I,\left(T_{i}, S_{i}, N_{S}\right)$ is a De Morgan triple, then $\left(T, S, N_{S}\right)$ is a De Morgan triple.

Proof. Since $N_{S}$ is the ordinal sums of $\left(a_{i}, b_{i}, N_{S}\right)$ then from Theorems 4.1 we have that $N_{S}(T(x, y))=S\left(N_{S}(x), N_{S}(y)\right)$.

If $(x, y) \notin\left[a_{i}, b_{i}\right]^{2}$ for all $i \in I$, then, for all $i \in I,(1-x, 1-y) \notin\left[1-b_{i}, 1-a_{i}\right]^{2}$ and therefore $N_{S}(S(x, y))=N_{S}(\max (x, y))=\min (1-x, 1-y)=T\left(N_{S}(x), N_{S}(y)\right)$.

If $x, y \in\left[1-b_{i}, 1-a_{i}\right]$ for some $i \in I$ then $S(x, y) \in\left[1-b_{i}, 1-a_{i}\right]$ and by Propositions 4.15, 4.16 and 4.17 (observe that $N_{S}$ also is the ordinal sums of $\left(1-b_{i}, 1-a_{i}, N_{S}\right)$ ), we have that

$$
\begin{aligned}
N_{S}(S(x, y)) & =a_{i}+\left(b_{i}-a_{i}\right) N_{S}\left(\frac{S(x, y)-\left(1-b_{i}\right)}{b_{i}-a_{i}}\right) \\
& =a_{i}+\left(b_{i}-a_{i}\right) N_{S}\left(\frac{\left(1-b_{i}\right)+\left(b_{i}-a_{i}\right) S_{i}\left(\frac{x-a_{i}}{b_{i}-a_{i}}, \frac{y-a_{i}}{b_{i}-a_{i}}\right)-\left(1-b_{i}\right)}{b_{i}-a_{i}}\right) \\
& =a_{i}+\left(b_{i}-a_{i}\right) N_{S}\left(S_{i}\left(\frac{x-a_{i}}{b_{i}-a_{i}}, \frac{y-a_{i}}{b_{i}-a_{i}}\right)\right) \\
& =a_{i}+\left(b_{i}-a_{i}\right) T_{i}\left(N_{S}\left(\frac{x-a_{i}}{b_{i}-a_{i}}\right), N_{S}\left(\frac{y-a_{i}}{b_{i}-a_{i}}\right)\right) \\
& =a_{i}+\left(b_{i}-a_{i}\right) T_{i}\left(\frac{N_{S}(x)-\left(1-b_{i}\right)}{b_{i}-a_{i}}, \frac{N_{S}(y)-\left(1-b_{i}\right)}{b_{i}-a_{i}}\right) \\
& =T\left(N_{S}(x), N_{S}(y)\right) .
\end{aligned}
$$


Theorem 4.3. Let $T, S$ and $N$ be the ordinal sum of the summands $\left.\left(1-b_{i}, 1-a_{i}\right), T_{i}\right),\left(a_{i}, b_{i}, S_{i}\right)$, $\left(a_{i}, b_{i}, N_{i}\right), i \in I$, respectively. If $N$ is a fuzzy negation and, for all $i \in I,\left(T_{i}, S_{i}, N_{i}\right)$ is a De Morgan triple, then $(T, S, N)$ is a semi De Morgan triple which satisfies Eq. (2.6).

Proof. Analogously from the Theorem 4.1.

Theorem 4.4. Let $T$ and $S$ be the ordinal sum of the summands $\left(1-b_{i}, 1-a_{i}, T_{i}\right)$ and $\left(a_{i}, b_{i}, S_{i}\right)$, $i \in I$, respectively. If, for all $i \in I,\left(T_{i}, S_{i}, N_{S}\right)$ is a De Morgan triple, then $\left(T, S, N_{S}\right)$ is a De Morgan triple.

Proof. Analogously from the Theorem 4.2.

\section{CONCLUSION AND FINAL REMARKS}

This work is a extension of the paper [20] presented in Fourth Brazilian Conference on Fuzzy Systems (IV CBSF), 2016. In this paper we consider the ordinal sum of the summands of a family of t-norms and t-conorms, and characterize the ordinal sum of the summands $\left(a_{i}, b_{i}, N_{i}\right)$ where $\left(N_{i}\right)_{i \in I}$ are a family of fuzzy negations and (]$a_{i}, b_{i}[)_{i \in I}$ a family of nonempty, pairwise disjoint open subintervals of $[0,1]$. In additon, we prove that the ordinal sum of the summands $\left(a_{i}, b_{i}, N_{i}\right)$ is a fuzzy negation. Finally, we prove if $\left(T_{i}, S_{i}, N_{i}\right)$ is a De Morgan triple satisfy some specific conditions, then $(T, S, N)$ is a semi De Morgan triple as well as if $\left(T_{i}, S_{i}, N_{S}\right)$ is a De Morgan triple, then $\left(T, S, N_{S}\right)$ is a De Morgan triple, where $N_{S}$ is a standard negation.

There are several types or extensions of fuzzy sets theory [8], among them is the fuzzy sets where the membership degrees are intervals proposed in an independent way in [23, 28]. As a future work, we will study ordinal sums of interval-valued De Morgan triples in the light of the interval representation ideas proposed in $[5,24,25]$ which was adapted for interval-valued t-norms, $t$ conorms and fuzzy negations in $[27,6,14]$. In addition, we will also investigate additive and multiplicative generators of (interval-valued) De Morgan triples.

\section{ACKNOWLEDGMENT}

This work is supported by Brazilian National Counsel of Technological and Scientific Development CNPq (Proc. 307781/2016-0 and 404382/2016-9).

RESUMO. Neste artigo, consideramos a soma ordinal dos sumandos das t-normas, $t$ conormas e negações fuzzy, e provamos alguns novos resultados sobre eles. Em particular, fornecemos algumas condições no sentido de garantir que quando os somandos forem triplas de De Morgan suas somas ordinais também são triplas de De Morgan. Também provamos que as leis de De Morgan são preservadas pela ação de automorfismos e N-dualidade.

Palavras-chave: t-norma, t-conormas, negação fuzzy, triplas de De Morgan, soma ordinal. 


\section{REFERENCES}

[1] C. Alsina, M.J. Frank \& B. Schweizer. "Associative functions - Triangular norms and copulas". World Scientific Publishing, Danvers, MA (2006).

[2] K.T. Atanassov. "Intuitionistic fuzzy sets, Theory and applications", volume 35 of Studies in Fuzziness and Soft Computing. Physica-Verlag, Heidelberg (1999).

[3] M. Baczyński \& B. Jayaram. "Fuzzy Implications”. Springer-Verlag Publishing, Berlin (2008).

[4] B. Bedregal, G. Beliakov, H. Bustince, T. Calvo, R. Mesiar \& D. Paternain. A class of fuzzy multisets with a fixed number of memberships. Information Sciences, 189 (2012), 1-17.

[5] B. Bedregal \& R. Santiago. Some continuity notions for interval functions and representation. Computational and Applied Mathematics, 32(3) (2013), 435-446.

[6] B.C. Bedregal. On interval fuzzy negations. Fuzzy Sets and Systems, 161(17) (2010), 2290-2313.

[7] G. Bezhanishvili, M. Gehrke, J. Harding, C. Walker \& E. Walker. "Varieties of algebras in fuzzy set theory". Elsevier B. V., Amsterdam (2005), pp. 321-344.

[8] H. Bustince, E. Barrenechea, M. Pagola, J. Fernandez, Z. Xu, B. Bedregal, J. Montero, H. Hagras, F. Herrera \& B.D. Baets. A historical account of types of fuzzy sets and their relationships, IEEE Trans. Fuzzy Systems, 24(1) (2016), 179-194.

[9] H. Bustince, P. Burillo \& F. Soria. Automorphisms, negations and implication operators. Fuzzy Sets and Systems, 134(2) (2003), 209-229.

[10] H. Bustince, J. Montero, E.B. M. Pagola \& D. Gomes. “A survey of interval-value fuzzy sets”. John Wiley \& Sons Ltd. (2008), pp. 491-515.

[11] T. Calvo. On mixed De Morgan triplets. Fuzzy Sets and Systems, 50(1) (1992), 47-50.

[12] C.D. Costa, B. Bedregal \& A.D. Neto. Relating De Morgan triples with Atanassov's intuitionistic De Morgan triples via automorphisms. International Journal of Approximate Reasoning, 52(4) (2011), 473-487.

[13] G. Dimuro, B. Bedregal, A.J. H. Bustince, M. Baczyński \& K. Mis. $Q L$-operations and $Q L$-implication functions constructed from tuples $(O, G, N)$ and the generation of fuzzy subsethood and entropy measures. International Journal of Approximate Reasoning, 82 (2017), 170-192.

[14] G. Dimuro, B. Bedregal, R. Santiago \& R. Reiser. Interval additive generators of interval t-norms and interval t-conorms. Information Sciences, 181(18) (2011), 3898-3916.

[15] J. Fodor \& M. Roubens. "Fuzzy Preference Modelling and Multicriteria Decision Support". Kluwer Academic Publisher, Dordrecht (1994).

[16] M. Gehrkea, C. Walker \& E. Walker. A note on negations and nilpotent t-norms. International Journal of Approximate Reasoning, 21(2) (1999), 137-155.

[17] E. Klement, R. Mesiar \& E. Pap. "Triangular norms”, volume 8. Kluwer Academic Publishers, Dordrecht (2000). 
[18] R. Lowen. "Fuzzy Set Theory: Basic Concepts, Techniques and Bibliography". Kluwer Academic Publishers, Dordrecht (1996).

[19] K. Menger. Statistical metrics. Proc. Nat. Acad., 28 (1942), 535-537.

[20] I. Mezzomo, B. Bedregal \& R. Reiser. New results about De Morgan triples. In "Proceeding of Fourth Brazilian Conference on Fuzzy Systems (IV CBSF)”, volume 28 (2016), pp. 83-93.

[21] H. Nguyen \& E. Walker. "A first course in fuzzy logic". Chapman \& Hall/CRC (2000).

[22] M. Öztürk, A. Tsoukias \& P.m. P. Vincke. "Preference modelling”. Schloss Dagstuhl, Germany (2006).

[23] R. Sambuc. "Fonctions $\phi$-floues. application l'aide au diagnostic en pathologie thyroidienne". Ph.D. thesis, University of Marseille, Marseille (1975).

[24] R. Santiago, B. Bedregal \& B. Acióly. Interval representations. TEMA - Tendências em Matemática Aplicada e Computacional, 5(2) (2004), 317-326.

[25] R. Santiago, B. Bedregal \& B. Acióly. Formal aspects of correctness and optimality of interval computations. Formal Aspects of Computing, 18(2) (2006), 231-243.

[26] B.B. Schweizer \& A. Sklar. Associative functions and statistical triangle inequalities. Publicationes Mathematicae Debrecen, 8 (1961), 169-186.

[27] A. Takahashi \& B.R.C. Bedregal. Interval t-norms, t-conorms, complements and implications. TEMA Tend. Mat. Apl. Comput., 7(1) (2006), 139-148.

[28] L. Zadeh. The concept of a linguistic variable and its application to approximate reasoning - I. Information Sciences, 8 (1975), 199-249.

[29] S. Zadroyzny \& J. Kacprzyk. Bipolar queries: An approach and its various interpretations. In "Proceeding of IFSA - EUSFLAT 2009, Lisbon, Portugal" (2009), pp. 1288-1293. 